\title{
DOCUMENTATION OF HISTORICAL BUILDING VIA VIRTUAL TOUR: THE COMPLEX BUILDING OF BATHS IN STRASBOURG
}

\author{
M. Koehl ${ }^{\text {a, }}{ }^{*}$, A. Schneider ${ }^{\text {b }}$, E. Fritsch ${ }^{\text {b }}$, F. Fritsch ${ }^{\text {a }}$, A. Rachedi ${ }^{\text {b }}$, S. Guillemin ${ }^{\text {a }}$ \\ ${ }^{\text {a }}$ Laboratoire ICube - UMR 7357, INSA de Strasbourg, F-67084 Strasbourg, France \\ (mathieu.koehl, samuel.guillemin)@insa-strasbourg.fr \\ b Service de l'Inventaire et du Patrimoine - DCTS - Région Alsace, F-67000 Strasbourg, France \\ (audrey.schneider, emmanuel.fritsch, florent.fritsch, abde.rachedi)@ region-alsace.eu
}

KEY WORDS: Documentation, Heritage, Virtual tour, Panorama, Historic building information system

\begin{abstract}
:
The virtual visits exist for several years and rest on open source or professional software packages allowing to realize complete animations. The historic buildings are often fragile, sometimes difficultly and only partially accessible. It is the complex case of the building of the municipal baths of Strasbourg, France, object of this study. It is thus interesting to use the technologies of the virtual visits to document a historic building. If we content ourselves only of panoramic images, the visitor cannot take completely advantage of the site, especially if he does not know it in advance. It is a question of proposing to the visitor a guided tour, constrained, allowing him to move on to all the recommended places. Then to supply him further information on the most significant parts and to propose him images of archive to make comparisons. Of course, if he wants to walk alone in and around the building, he will have the leisure of it, but at his own risk. To realize such a visit, the paper shows the various necessary stages of elaboration, in particular by beginning with the writing of a scenario of the visit. This project written in several hands allowed to combine the knowledge of diverse actors working in the field of the inventory and of the heritage valorisation.
\end{abstract}

\section{INTRODUCTION}

\subsection{Context}

The historic buildings are a part of inescapable elements in the cultural aspects of a country. Some particular buildings had specific roles in a time not so distant and continue in their function even today. It's the case of the municipal baths of Strasbourg, continually and currently in service. But as it is about particular buildings, their visit, their interpreting and their access is not any more very well-to-do. The idea of the implementation of a guided virtual visit was born to be able to make, remotely, the visitors of the deprived access benefit to these buildings. This paper aims at describing the various stages of the design, the acquisition then the implementation of this guided virtual visit. In main stages we so find i) the writing of a scenario of guided tour, ii) the realization of shots of photographs, iii) the calculation of the panoramic images, iv) the conception of texts and accompanying documents, v) the choices of the symbolism (avatar, points of view, information) and finally the calculations of the definitive virtual visit to be able to spread them on an interactive terminal and on a web site of Department of the Inventory and the Heritage of the Region Alsace, France. This visit was thus written in several hands, by combining the skills of photographers, topographers, historians and specialists of the inventory and the heritage in decorations and in architecture. This project was realized within the framework of the valuation of "Neustadt" of Strasbourg, as historic district candidate in the UNESCO world heritage (Strasbourg.eu, 2013). The "Neustadt" appears among the urban extensions the most remarkable and best kept by the bend of the XIXth and XXth centuries in Europe. The candidacy to the inscription on the UNESCO world heritage list aims at making recognized this exceptional inheritance. The virtual visit was realized in particular for an animation during the "Days of the Neustadt" (Région Alsace, 2013).

\subsection{History of the Municipal Baths of Strasbourg}

If the idea of the creation of a complex building of baths in Strasbourg sprouted from 1891, it was more seriously considered in 1894 by the City Council, chaired by mayor Otto Back, with the goal to improve the health service of a city in full population boom. A first project designed by the chief architect of the City, Johann Karl Ott, was presented in 1901. But it was the project developed by Fritz Beblo, appointed municipal architect and inspector of the buildings of Strasbourg in 1903, assisted by the architect Ernst Fettig, which was, at least, chosen in 1904. The project took into account the design of the whole former project of Ott.

The building had to contain besides a popular municipal bath, a therapeutic section with medicinal baths, massage rooms and a private dental hospital.

To bring the construction to a successful work, the "Strassburger Baugesellschaft A.G" has been endowed with an estimated 820000 marks envelope from 1904. The construction was led from 1905 till 1908. The building, of a total surface of 4765 square meter, was able to open its doors on August of the same year.

Very fast, the inhabitants enjoyed the benefits of sunbathing on the terraces of the solarium, but especially the therapeutic virtues of "irish-russo-roman" baths. This treatment, mixture of three traditions, was recommended as well to sick as to healthy people as a precautionary measure. It consisted in alternating

\footnotetext{
* Corresponding author.
} 
the steam room with hot air, the shower, massages, the steam bath, then three baths in swimming pools: the warm $\left(36^{\circ} \mathrm{C}\right)$, the so called "Wildbad" $\left(34^{\circ} \mathrm{C}\right)$ and the cold $\left(21^{\circ} \mathrm{C}\right)$, to finish by a hot and cold shower and a compulsory rest of half an hour on deckchairs. Shortly after the opening of the baths, the authorities committed the second part of the project, that of the medicinal baths at the end of 1908 . These works were finished in 1910 and the operational medicinal structures in July, 1911. This new extension included installations of mechano-therapy, hydroelectric baths, fango-therapy (mud baths), a Swedish gym room, suffer baths and the office of the bathing doctor. The private dental hospital was installed on the second floor in 1911.

Nowadays, only both swimming pools, steam rooms with overheated and steam air and the Roman baths remained intact, with their original taps for the main part. The medicinal baths worked under diverse forms until 1974.

The Fig.1 shows the main frontage of the Baths of Strasbourg.

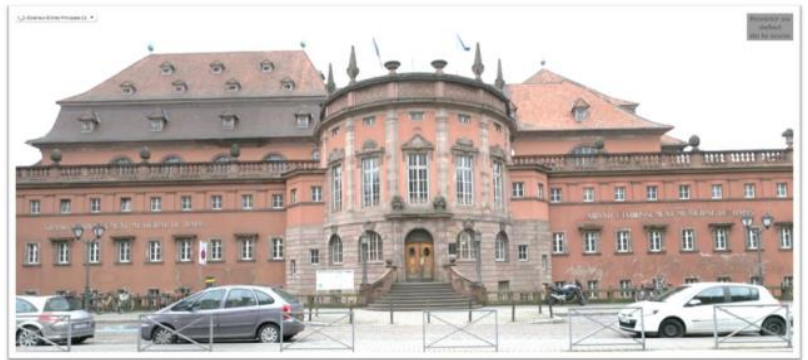

Figure 1: Main façade and entrance of the Baths of Strasbourg

\section{RELATED WORKS}

\subsection{Virtual tours of historical buildings}

One of the symbolic buildings illustrated by a virtual visit and having in more a pronounced cultural character is the one of the Louvre Museum (Paris, France) (Louvre, 2013). It is not so much a question of discovering the building, but rather the works which are exposed to it, what also corresponds to one of the aspects looked for in our visit of the baths of Strasbourg. The visit consists of a web site containing 360 degree photographic panoramas. An overall map allows to find the way in the place, he indicates the various available points of view in the visit. This visit also contains links towards internet pages presenting further information on the various works as well as the possibility of seeing certain works in close-up.

The virtual visit of Oxford (Oxford, 2004) is also very famous. This visually stunning resource uses interactive web pages which allow to explore and to manipulate 360 degree photographic panoramas of the city and university. It is constructed from hundreds of high quality photographs of Oxford. Highlighted in the Sunday Times in January 2004.

There exist a photography tutorial blog about how these panoramas were captured and created (Harrisson, K., 2006).

Another interesting and world project is the one launched recently by the Google foundation: it is about the World Wonders Project (WWP Google, 2012). 130 historic sites in more than 18 countries are documented under the shape of a website proposing besides panoramic views, information under the shape of a descriptive index sheets, videos of users and more rarely 3D models. By using the Street View technology, Google has a unique opportunity to make world heritage sites available to users across the globe. Street View is a hugely popular feature of Google Maps which is already available in dozens of countries. It allows users to virtually explore and navigate a neighborhood through panoramic street-level images. With advancements in its camera technologies Google can now go off the beaten track to photograph some of the most significant places in the world so that anyone, anywhere can explore them.

Street View has already proved a real hit for tourists and avid virtual explorers. The World Wonders Project also presents a valuable resource for students and scholars who can now virtually discover some of the most famous sites on earth. The project offers an innovative way to teach history and geography to students all over the world. Our World Wonders Project is also supported by a broad, connected suite of other Google technologies, bringing wonders of the world within reach of an unprecedented global audience. The project website also provides a window to $3 \mathrm{D}$ models, YouTube videos and photography of the famous heritage sites. Together with partners including UNESCO, the World Monuments Fund and Cyark, the World Wonders Project is preserving the world heritage sites for future generations.

There is in France also a directory of the French-speaking archaeology (Archéophile, 2013) which lists a multitude of sites proposing virtual visits, located in particular in France. But we also find equivalents in other numerous countries.

\subsection{Software for panoramic production and virtual tour edition}

There is a lot of software you can use for the realization of the virtual visits. If you want to be able to produce stunning imagery, Adobe Photoshop (Adobe, 2013) is an absolute must. Photoshop will also stitch panoramic images together with a nice clean result. It is also by far the simplest method for producing panoramas. On the flip side however, it completely handles the stitching and color blending process and has no tool for editing files in intermediate states. You basically just ask it to process and either like or dislike the result.

Autopano Pro or Autopano Giga (Kolor, 2013): this is by far the best and most efficient way to create panoramic images. It has all the bells and whistles allowing you to fully edit and every aspect of the project. It allows you to view a preview of the panorama before you commit to rendering. Unlike Photoshop, Autopano allows you to tweak every aspect from layers and color correction to vertical and horizontal line correction. It also supports multiple exposure brackets!

The virtual site of the (French Ministry of Culture, 2013) allows to discover its buildings and its inner courtyard. The used technology is based on the krPano library (krPano, 2013). One of the proposed originalities is the switching possibilities between the view in the daytime and in the night view. Numerous hotspots allow to reach pages containing complementary information, images of archive or still to change to another panorama. Some limits of zoom are not set what may head the visitor for very improbable visualization modes and displays from where it is not very easy to stand out.

The virtual visit of the building of the World Trade Organization (WTO, 2013) allows to introduce another aspect of the virtual visits, in particular the proposed environment of visualization. In this case, the viewer is supplied by Pano2VR (gnomeSoftware, 2013). The export formats mainly used are spherical or cylindrical panoramic images into Adobe Flash 10, HTML5 (WebGL / iPhone / iPad), or QuickTime VR (QTVR) with features such as customizable skins, multi-resolution (Giga-pixel panoramas), hotspots and directional sound. 
We find the same features under Panotour pro (Kolor, 2013). It is this last software package which was used for the creation of the definitive virtual visit in Adobe Flash format.

\section{STEPS FOR VIRTUAL TOUR DESIGN}

\subsection{Context}

As a masterpiece of the "Neustadt of Strasbourg", the complex building of baths of Strasbourg has been selected for the realization of a historic documentation in form of a virtual tour. This tour has been designed as a guided tour with the main goal to allow the public to follow a predefined scenario for visiting virtually the emblematic building.

This tour contains more than 46 stages marked out by numerous $180^{\circ} \times 360^{\circ}$ panoramic images, many large panoramic images and some small panoramic images used for transitions.

The phase of recording has been preceded by a phase of conception allowing defining the best points of view and the route of the visit.

\subsection{Writing of a scenario of guided tour}

The first stage of this project consisted in defining a very detailed visit of the building accompanied by the manager of the institution as well as specialists of the inventory and heritage in particular as regards the architectural and decoration parts of the building. It is moreover about these two main aspects which the virtual visit will have to allow to emphasize and to show remotely to the virtual visitors.

At the end of this visit, we were able to proceed to the development of the main scenario of the guided tour. It is indeed about a guided tour, thus constrained, which has to allow a visitor who didn't know the building to walk there virtually but especially make it the complete tour by avoiding bends and impasses. The scenario is built on the basis of the maps of the building. From the beginning of the project, the virtual visit owes to be complete and to not contain white or evaded zones. The visit will thus contain spherical panoramic images in the main places, but also simple panoramic images to indicate the transitions, the passages of sieves, gone up by staircases, for example.

The Figure 2 represents the map of the complex of buildings on which was indicated the main progress of the guided tour. Three floors of the building are represented on the same view; the use of colour allows to indicate the corresponding floor. This overall map is reused in the visit virtual as situation map. The positions of points of view are made interactive and allow to reach directly places chosen by clicking the overall map. This mode of browsing is recommended only for advanced visitors, knowing the site and looking for different things than a guided tour, because the thread of the visit is lost by following this process.

The script allows to delineate the virtual guided tour. For every documented place, the scenario will also allow to leave towards all the related places but by going away from the guided tour. This option will allow, even there, a visitor experimented to move as he wants in the building, or possibly to another visitor to retrace his steps to expand the visit. This possibility of visit out of the guided route requires the duplication of images panoramic as we are going to detail it in the section 3.5.

The following stage consists in taking photographs allowing then the calculation of the panoramic images in spherical projection.

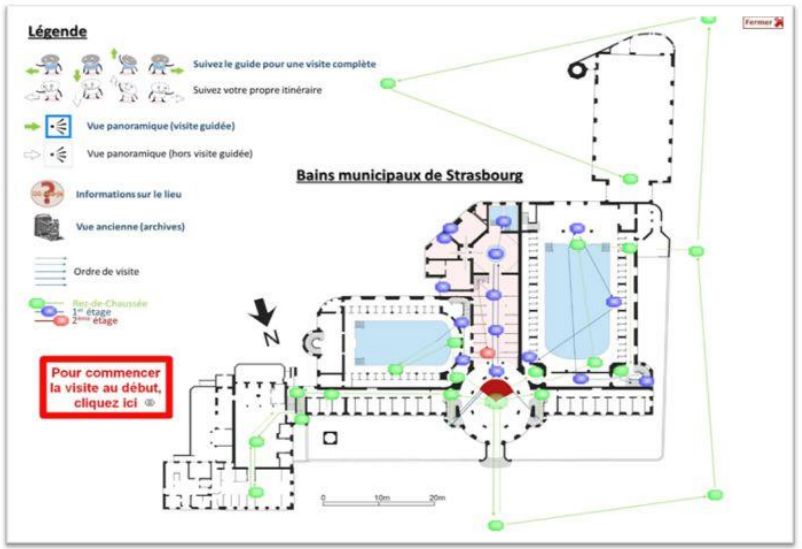

Figure 2 : Main map and tour scenario

\subsection{Shots and realization of the panoramic images}

For each point of view of the scenario, the parameters of photographs have been predefined then recorded during the field acquisition and finally used during the image processing. The panoramic images have been realized by using a Canon EOS 5D camera. The shooting was to be taken to allow the assembling of series of 49 high-resolution photographs. A minimal recovery of $25 \%$ to $30 \%$ was taken into account to enable the processing software to combine the pictures into one panorama. A tripod and a panoramic head device to eliminate any parallax between two photographs have been used. The panoramic head Manfrotto 303 allows, thanks to its notching, placing the unit in a same position, allowing taking photographs again with different settings. It is essential to make use of a levelling unit so that the camera remains horizontal during rotation, in order to avoid a cropping of the photographs.

Shots have been taken in natural lighting, what has not facilitated the acquisition, but has allowed to restore the particular atmosphere of this building (Fig.3).
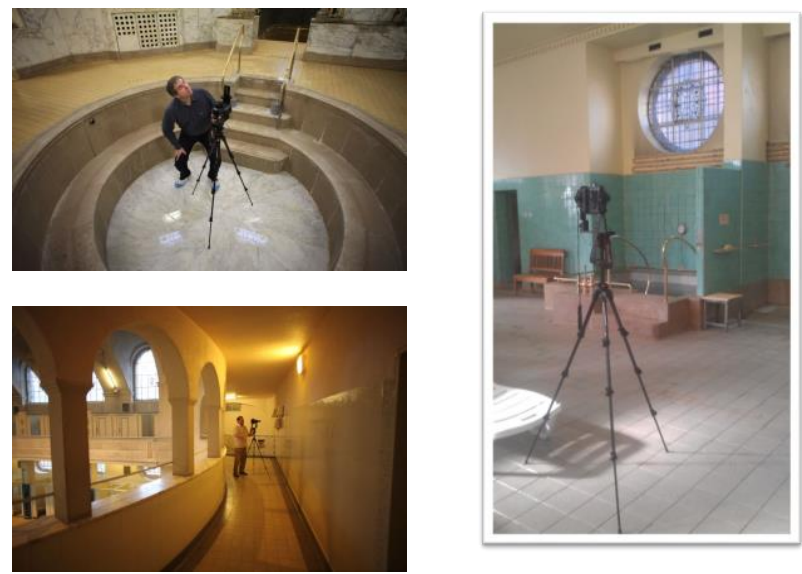

Figure 3: Shooting of photographs in interior lighting conditions

The assembly and photo rectification was performed on the Autopano Giga (Kolor, 2013) software. While a recording in optimal conditions requires approximately 8 minutes per series of photographs, an automatic calculation of reorganization of the spherical panoramic image takes in average the same computer time (with optimal photographs) (see Fig.4). 


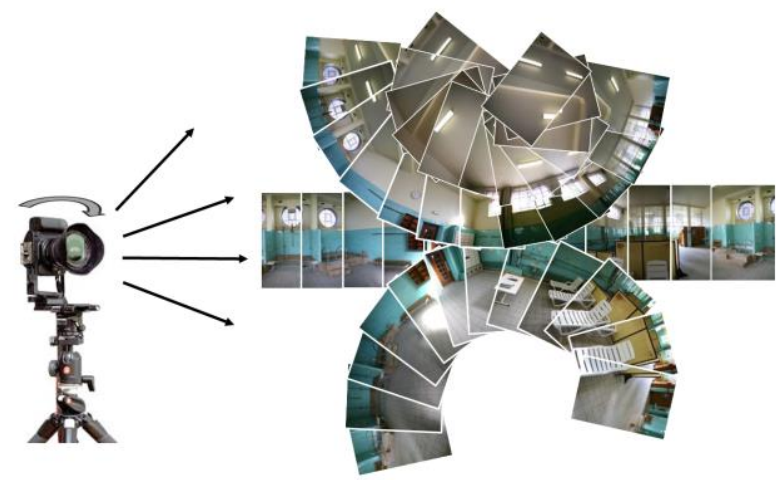

Figure 4: shooting of 49 photographs for composition of panoramic image.

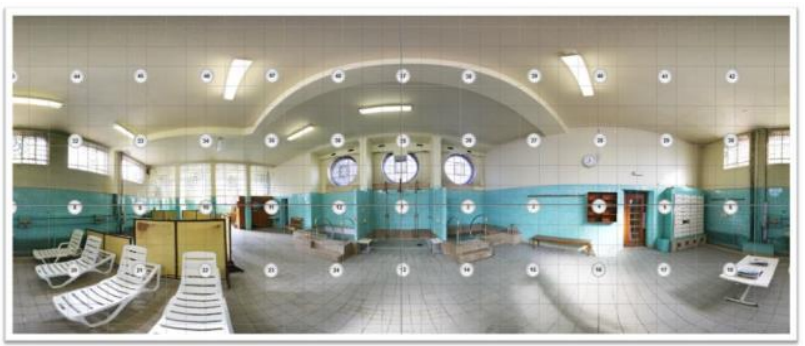

Figure 5: Panoramic composition in spherical projection calculated with Autopano Giga tool (Kolor).

The main visit contains five main places: the visit begins with the main entrance in the form of rotunda with two levels. It continues by the big swimming pool which is documented by two points of view at the ground floor and three points of view at the level of the gallery of the first floor. We borrow then a passage towards the small swimming pool. There a point of view from the gallery of the first floor allows then to return towards the edge of the pond ( 2 additional points of view).

The visit joins then the main entrance for the second time. Two staircases on both sides allow then to go up on the first floor where we find the Roman baths. These contain secondary rooms with tepid and cold baths, massage room, two steam rooms with hot air and very hot air and of course individual cabins. Here we find 4 spherical panoramas.

The visit continues then by the medicinal baths, then after return again by the main entrance, the tour continues outside around, with 5 points of view.

The tour finished by a view located in the annex of the boiler room, not interesting from the historic point of view but impressive from the point of view of the machinery.

The calculated panoramas gives rise to images of about 48.3 MB with average resolutions of $11472 \times 4944$ pixel. The images are processed to homogenize their luminosity and contrast. Indeed, working in natural light, and in artificial light and in $360^{\circ}$, certain parts will be necessarily against the light. A preprocessing of the images, taken care by the software of stitching allows this equalization. A catching up by image processing after calculation is always possible. Another difficulty is the one concerning regular motives. Indeed, for the constitution of the panoramic image, the software looks for homologous points in the images by basing itself on peculiarities. In the case of objects presenting regular wefts, the software can be easily misled and associate false images. The fact of using the panoramic head and of acquiring the images in the form of a well determined sequence allows to reconstitute manually the panoramic image by knowing the rough position of the centres of the images. The software can calculate then more easily the final panoramic image by taking into account these new constraints. While an image processed in an automatic way is generated within 10 minutes, the manipulation and the manual positioning of every image, then the calculation by taking into account these new constraints will take approximately 3 times the initial time. Finally, a quality indicator of the links between the various images allowing the reorganization of the final panoramic image will insure us of a good quality of panoramic image (Fig. 6).

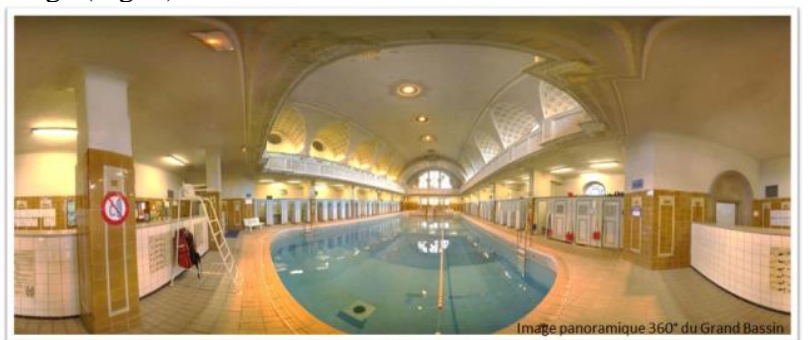

Figure 6 : Panoramic image of the big pool seen from the beach on the north side
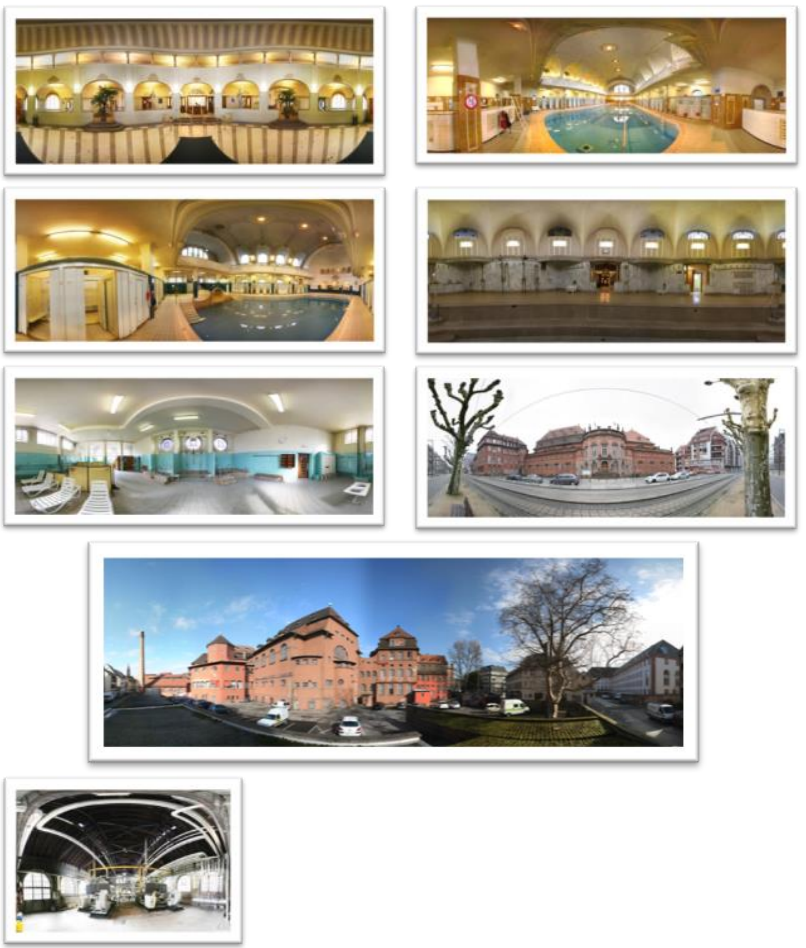

Figure 7: Main panoramic images

\subsection{Texts and accompanying documents}

In the various examples quoted in the similar works we were able to find out that the purpose was not only to create a virtual visit, but that such a virtual visit can be considered as an interface of an architectural and historic system of knowledge. This kind of interface can be obtained by adding links (hotspots). These links can point towards an URL address, towards a HTML page, towards a digital document or allow to pass by using a transition from a panoramic image to another one. To feed the database of knowledge, the members of the project developed information sheets on the elements of decorations on one hand and on the architectural elements on the other hand. During the visit, these elements are accessible by simple click, they are identified in the panoramic images by 
small descriptive signs like information panels. These information sheets can be added according to the interpretation and to the valuation of the site. A simple recalculation of the final virtual visit will be necessary. Dynamic links could be also added or created and associated to HTML pages. This had already been successfully experimented in (Koehl et al., on 2012).

Figure 8 shows two types of texts of information associated to the particular elements of the visit virtual.

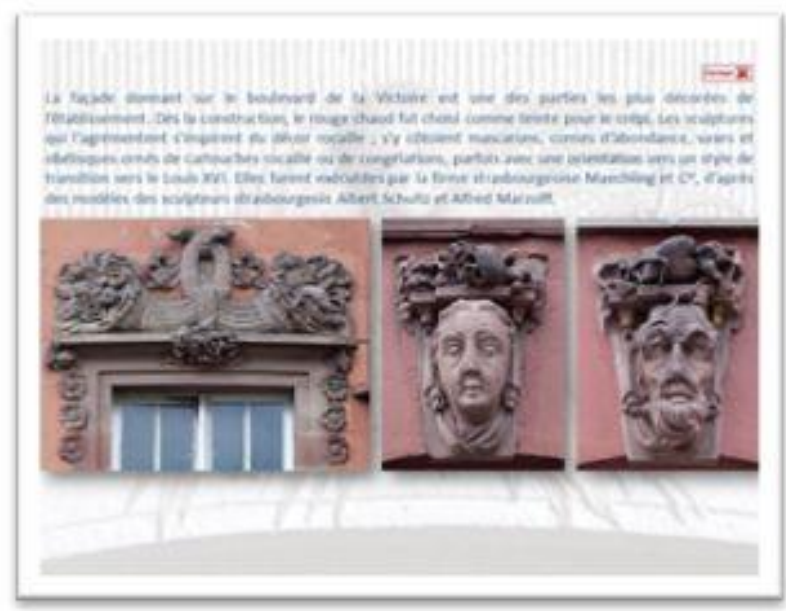

Figure 8: Complementary information sheet.

As other elements, we add also gladly archives images allowing to make the link between past and building in its current state (Fig.9).

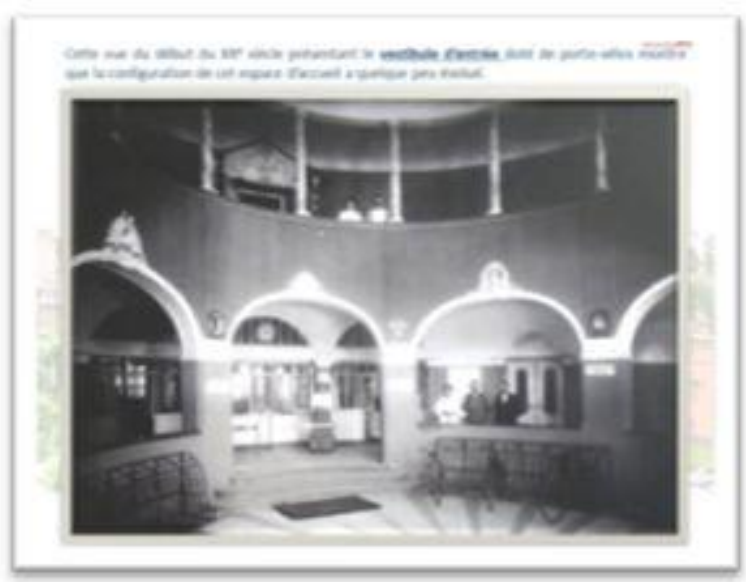

Figure 9: Image of archive integrated in the virtual tour.

\subsection{Avatar and peculiarities of the virtual tour}

To make the visit more intuitive, because let us remind that it is a guided tour, we added a particular signalling system. Already evoked for the access to further information, this signalling system allows to visualize the various accessible points of view out of the guided tour. An avatar was also created. In colour, it indicates the path of the guided tour, in black and white, it indicates all the possibilities of routes by going away from the initial path (Fig. 10).

A help page, accessible at any time and especially in the beginning of visit also allows to verify the manipulations of the mouse or on a touch-sensitive screen.

Another peculiarity of this guided visit is the multiplicity of the proposed visit routes. Indeed, a precise signalling system allows to indicate the official route. But at any time, the visitor can go away from the guided tour and decide to display panoramas in a different order. Technically, this requires the duplication of most of the panoramas in the design of the virtual visit. Indeed, to follow the constrained succession of the panoramas the user has to follow a coloured avatar; but if the user wants to go away from the guided tour, identical duplicated panoramas have to allow transitions towards all the accessible panoramas from the place in question and that without proposing one more particularly. In these panoramas the avatar is in black and white. The duplication of the panoramas is relatively easy, it increases simply the calculation time during definitive generation of the tour described in the following section.

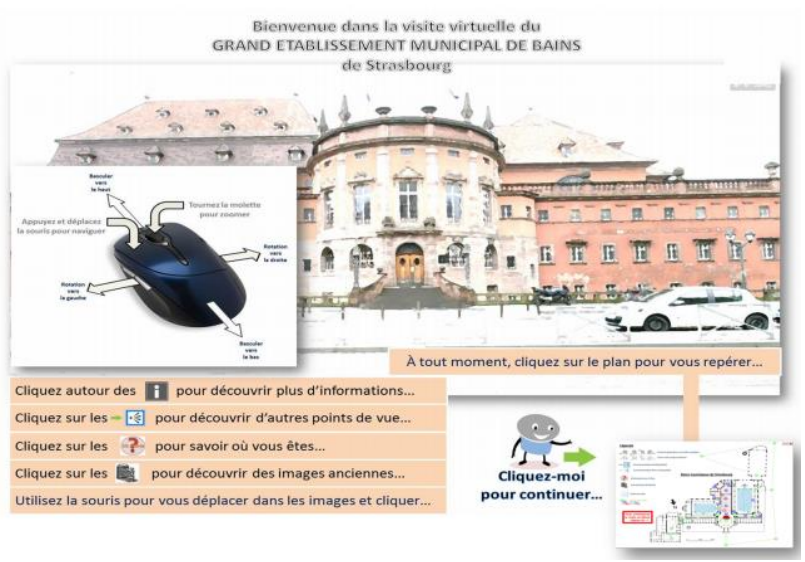

Figure 10: Avatar for the path of the guided tour.

\subsection{Definitive calculation of the guided tour}

The last phase of the design of the virtual visit consists in renewing of the original scenario basing itself on the panoramic images and on the images of transition now definitively calculated. For every image it was a question of selecting and defining the different types of following elements: i/ hotspot allowing to pursue the visit towards the following panoramic image and according to the path of the guided tour, ii/ hotspot allowing to return to the previous stage, iii/ hotspots towards all the images connected with the active image but out of the guided tour, iv/ regions allowing to display in detail professional images of very high-resolution, v/ links towards comments, explanatory texts, historic documents, photographs and images of archives, etc.

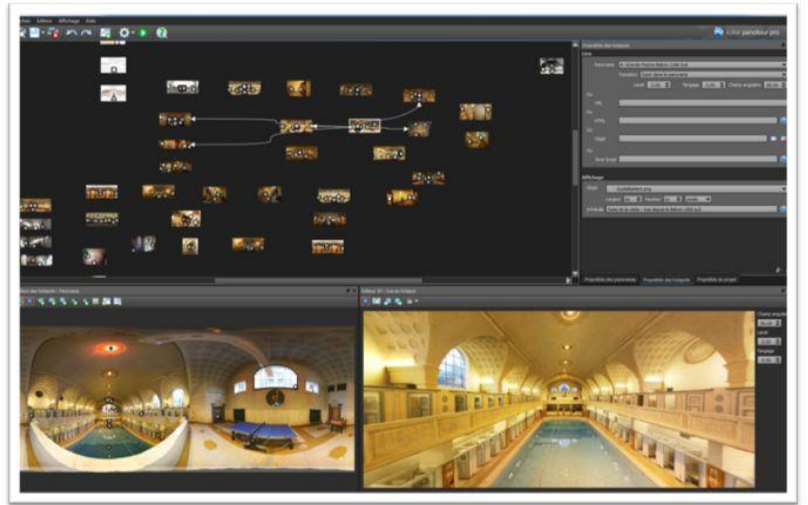

Figure 11: Panotour pro (Kolor) GUI for tour calculation

For every panoramic image the initial parameters have been defined and also the values or the parameters fixing the limits of navigation and zoom, etc. Avatars have been created to identify 
the direction of main route of the guided tour. Particular pictures have been created to arouse an intuitive navigation, because this one was intended for novices not necessarily wornout to the digital technologies or 3D navigation (Fig.11).

Finally the virtual visit was realized by using the Panotour Pro (Kolor) software environment and tools. It appears in the form of an animation directly viewable as Adobe Flash in a Web environment.

\section{FURTHER EXPERIMENTATIONS}

A first scan by means of a Faro Focus 3D TLS was also undertaken and integrated - in the form of video - into the virtual visit. A 3D modelling can now be envisaged for the most significant parts of the buildings.

Besides the documentary value of the panoramic images, the complements brought by the connected documents allow at different levels, from the simplest reader to the historian and scientist to walk virtually in this building, to visualize the slightest details, to consult a rich associated documentation and, at least to discover the contents of this architectural jewel and its composition completely dedicated to the well-being of the inhabitants of Strasbourg. This paper shows the different steps of the creation and how the virtual tour can be used as an animated interface of a historical documentation system.

\section{CONCLUSION}

This project allowed to reflect and to set up a constructed process of definition of guided virtual visit of a historic building, and, in the same time, to propose it in the discovery for possibly distant visitors, but also to enrich this visit in historic information on the architecture, decorations and use of this one in time. The elaboration of 360 degree panoramic photographs was also able to be experienced and the parameters of recording refined in particular in the case of shots of inside and in natural or artificial light. This experiment can be used within the framework of next more and more complex projects of documentation associated with more structured knowledge bases.

\section{References:}

Adobe, 2013. Adobe Photoshop for creating HDR panoramas. http://www.adobe.com/en/products/photoshop.html?

Archéophile, 2013. Annuaire de l'archéologie française. http://www.archeophile.com/rwcat_168-visites-virtuelles.htm gnomeSoftware, 2013. Conversion software. http://gardengnomesoftware.com/pano2vr.php Google WWP, 2012. Google's World Wonders Project. http://www.google.com/intl/fr/culturalinstitute/worldwonders/

Harrisson, K., 2006. Panorama Tutorials by Dr. Karl Harrisson. http://www.chem.ox.ac.uk/oxfordtour/Tutorial/index.asp?ID=51 \&pagename $=$ Abode $\% 20$ Flash $\% 20360 \% 20$ Panoramas

Koehl, M., Brigand, N., 2012. Combination of virtual tours, 3D model and digital data in a $3 \mathrm{D}$ archaeological knowledge and information system. In: IAPRS, Vol. XXXIX-B4, XXII ISPRS Congress, 25 Aug.-1 Sept. 2012, Melbourne, Australia, pp. 439444.

Kolor, 2013. The best solutions for $360^{\circ}$ and panoramic imagery.

http://www.kolor.com/en

krPano, 2013. Small and high-performance viewer.

http://krpano.com/

Louvre, 2013, Visite virtuelle du Louvre, Paris, France.

http://www.louvre.fr/visites-en-ligne

http://musee.louvre.fr/visite-louvre/index.html?

Ministère de la Culture, 2013. Visite virtuelle du Ministère de la Culture Français.

http://www.visite.culture.gouv.fr/\#/fr/annexe/intro/t=Minist $\% \mathrm{C}$ 3\%A8re\%20de\%201a\%20Culture\%20-\%20Visite\%20virtuelle

OMC, 2013. Visite virtuelle du bâtiment de l'Organisation Mondiale du Commerce.

http://www.wto.org/images/panorama/Front.swf

Oxford, 2004, Virtual tour of Oxford

http://www.chem.ox.ac.uk/oxfordtour/

Région Alsace, 2013. Service de l'Inventaire et du Patrimoine. http://patrimoine.region-alsace.eu/

Strasbourg.eu, 2013, http://www.strasbourg.eu/developpementrayonnement/metropole-culturelle/patrimoine-culturel/strategiepatrimoniale/reconnaissance-patrimoniale-neustadt/candidatureneustadt-patrimoine-mondial-unesco

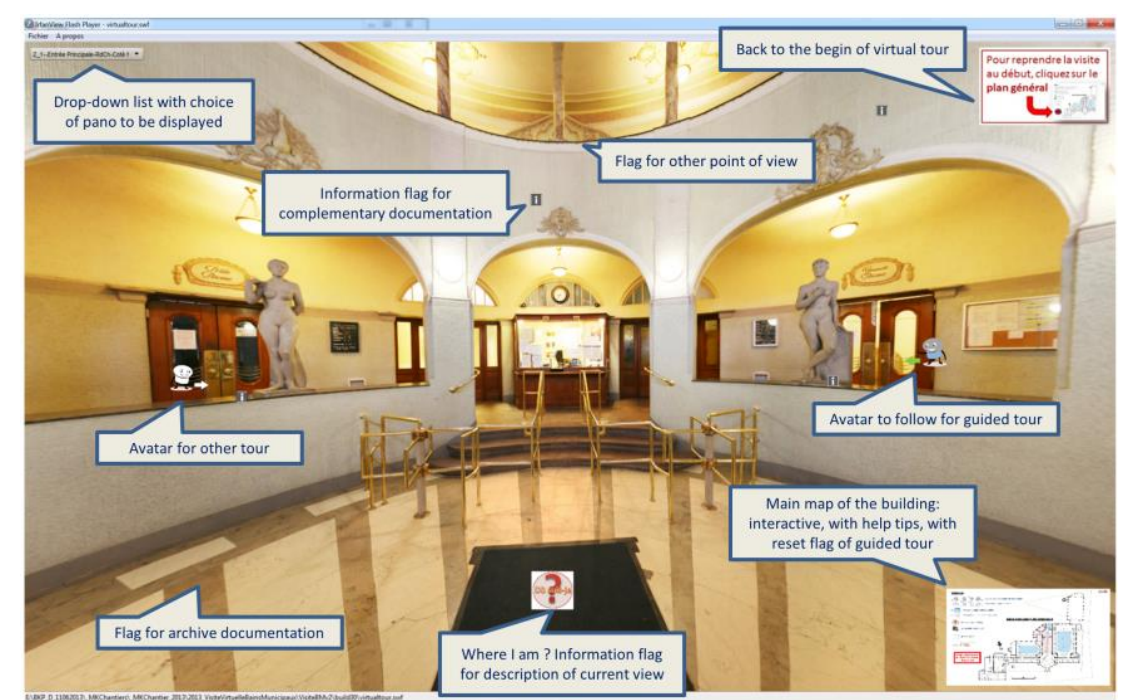

Figure 12: Main entrance of baths of Strasbourg with tags and different options for navigation and knowledge 